\title{
El escrito autoetnográfico: una propuesta en el ámbito universitario
}

\section{Resumen}

$\mathrm{E}$ n los últimos años, he observado que las competencias de lectura y escritura académicas han comenzado a tener un papel sustancial en la formación del profesional universitario para el diseño y composición de géneros textuales de su ámbito. A su egreso, se le exige amplios conocimientos de la disciplina y, a la vez, de profundas capacidades para la comunicación oral y escrita. Percibo que la cultura universitaria se muestra poco permisiva a innovaciones curriculares que las fomenten. Enfrenté mi realidad a partir de la asignatura de Ensayo científico, mediante una propuesta de intervención educativa, al amparo del enfoque género-proceso [Process-Genre Approach (PGA), por sus siglas en inglés], con estudiantes del grupo $2^{\circ} \mathrm{B}$ de la carrera de Médico Cirujano, bajo la interrogante "¿qué actividades pueden fortalecer sus habilidades y destrezas hacia la lectura y escritura académicas?", a partir de la cual realicé diversas transformaciones curriculares. Los trabajos finales entregados - no exentos de errores-, el acompañamiento y las vivencias, mostraron resultados aleccionadores.

Palabras clave: escritura académica, estudiantes universitarios, intervención educativa.

\section{Introducción}

La Escuela Superior de Medicina (hoy Unidad Académica de Medicina) (UAM, 2019) de la actual Universidad Autónoma de Nayarit (UAN) abre sus puertas en el mes de marzo de 1974 y, con ella, se institucionaliza la enseñanza de la medicina. Al paso de los años, ha experimentado diversas transformaciones: su programa de estudio $-\mathrm{A}$ 36- cambió a Competencias Profesionales Integradas; se rehabilitó y amplió su planta física; ha ido cultivando su prestigio al interior de la sociedad, en parte, por la acreditación de sus programas de licenciatura y posgrado.

Inicié mis actividades docentes en la UAM dos años después de su fundación. En aquellos tiempos, el Programa de Medicina General Integral (A-36), a pesar de sus innovaciones, no hacía un especial énfasis en el fomento a las habilidades de lectura y escritura académicas (LYEA); se daban por hecho algunas de nuestras concepciones actuales: esas habilidades deberían estudiarse en ciclos anteriores o eran contenidos de cursos de lectura y redacción, alejados de la disciplina e, incluso, "recaían en el ámbito de la responsabilidad de los padres de familia" (Carlino, 2005: 18).

Al paso de los años, este panorama empezó a tener grandes cambios. En los últimos tiempos, he observado que las competencias de LYEA -“[...] nociones y estrategias para participar en la cultura discursiva $[\ldots]$ como en las actividades de producción y análisis de textos [...]"(Carlino, 2005: 13) intentan tomar un papel sustancial en la formación del profesional universitario, pues se les pide tener amplios conocimientos de la disciplina como también capacidades para la comunicación oral y escrita, las que empatan con la actual realidad laboral, incierta, compleja y altamente competitiva. Este requerimiento también se equipara con las diversas dificultades que muestran y expresan los estudiantes al momento de escribir sus diversos trabajos finales y la ausencia de apoyos institucionales al respecto. Los testimonios se encaminan a diversos aspectos: ortográficos, sintácticos, estructurales (coherencia, cohesión), citación correcta de los autores en el texto y problemas de plagio. Todo ello en un contexto universitario nayarita, poco amigable para apoyar innova- 
ciones curriculares que las revitalice, generalmente en un ambiente muy árido.

En este contexto, ¿qué importancia se les da a las competencias sobre la LYEA? Por el momento, las circunstancias nos dicen que no es un tema institucionalmente prioritario: sólo 3\% de las asignaturas del plan de estudios -Desarrollo de habilidades del pensamiento y Ensayo científico- atienden estas competencias, las cuales, en opinión de muchos estudiantes, son de "relleno". Esta visión estudiantil muestra que las materias propias de la disciplina se comportan como esos "dispositivos de control" (Chartier, s.f.) que expresan relaciones de poder entre los saberes (vale más el examen de fisiología o bioquímica que el ensayo sobre la historia clínica, la autobiografía o la monografía); en el perfil de ingreso hay una mención tangencial al respecto: "Dominar los conocimientos básicos de física, química, matemáticas, biología y español" (UAM, 2019); en el perfil de egreso no hay referencia alguna.

En el aula, la enseñanza cotidiana de la disciplina es mayormente expositiva: uno puede observar a los estudiantes presentando en equipo un tema y al maestro escuchando; las libretas, cuadernos, lápices y bolígrafos empiezan a ser cosa del pasado, se han sustituido por la tablet o la computadora personal; se da el caso extremo cuando, en lugar de escribir en su cuaderno, el estudiante enfoca la cámara de su celular y toma una fotografía de la imagen proyectada. Es este cambio profundo de las herramientas de escritura que estamos viviendo, como lo dice Chartier (s.f.): leemos más y escribimos menos. Por lo que concierne a los trabajos escolares (resúmenes, ensayos, bitácoras, monografías), se les otorgan los porcentajes más bajos en la calificación final; algunas situaciones que observamos es que se solicitan, pero el docente no orienta (asesora) en su elaboración; no se regresan a los estudiantes, por lo que desconocen si tuvieron o no errores y de qué tipo, y cada fin de semestre encontramos "pilas" de trabajos finales engargolados en los escritorios de los docentes, disponibles para ser utilizados como material reciclable.

\section{Desarrollo}

Confieso nunca haber escuchado la palabra autoetnografía. Fue hasta el $18^{\circ}$. Seminario de Investigación organizado por la Universidad Autónoma de Aguascalientes (2017), cuando escuché la exposición: "La autoetnografía como estrategia de investigación para abordar temáticas relevantes para estudiantes de nivel medio superior". A partir de ese momento realicé una búsqueda de autores en los sitios de Google Schoolar, Redalyc, Scielo y Conricyt (Consorcio Nacional de Recursos de Información Científica y Tecnológica). Tuve mi primer encuentro con los escritos de:

- Mercedes Blanco: “¿Autobiografía o autoetnografía?"

- Ellis Carolyn, Adams Tony E. \& Bochner Arthur P.: "Autoetnografía: un panorama"

- Montagud Mayor Xavier: "Analítica o evocadora: el debate olvidado de la autoetnografía"

- Méndez Mariza: "Autoethnography as a research method: advantages, limitations and criticisms"

- Mercedes Blanco: "Autoetnografía: una forma narrativa de generación de conocimientos"

- Custer Dwayne: "Autoethnography as a transformative research method"

- Heewong Chang: "Autoethnography as method"

Estos antecedentes fueron el motivo principal para la selección de la autoetnografía como el escrito final, que tiene en mente a la escritura como un método, "el escribir en primera persona y traer la emoción y la subjetividad" (Bochner \& Ellis, 2016: 11); también pensé en los propósitos para su elaboración que, mediante ella, "el escritor hiciera uso de su experiencia personal para explorar sus prácticas culturales" (Ellis, 2005; Goodall, 2000, citados en Pelias 2003: 384); es decir, que combinara el análisis cultural y la interpretación con los detalles narrativos; "que los escritos reflejasen el análisis y la interpretación dentro del amplio contexto sociocultural" (Chang, 2008: 46), a la vez de lograr diversos beneficios: quebrantar las normas de la práctica de la investigación y la representación; trabajar desde dentro del conocimiento; maniobrar a través del dolor, confusión, enojo y la incertidumbre, y hacer una vida mejor rompiendo el silencio y reclamando la voz y el escribir, porque cuando se escribe una autoetnografía, se estudia y escribe la cultura desde el yo, "se mira hacia adentro (identidades, pensamientos, sentimientos y experiencias) y hacia afuera (en nuestras relaciones, comunidades y culturas)" (Holman, Adams \& Ellis, 2014: 46).

Un elemento que incorporé para despertar el interés estudiantil fue que la autoetnografía recuperara las experiencias que hasta el momento les ha dejado la asistencia a los espacios clínicos durante el pasado y el presente semestres, así como su impacto hacia su formación profesional. Las prácticas clínicas, desde la década de los setenta, han sido un elemento de formación sustancial, a las cuales se les dedica un promedio de cuatro a cinco horas por 
semana. Su propósito es claro: vincular teoría y práctica, conocer el funcionamiento del sistema de salud mexicano por medio de las clínicas y centros de salud, destacar el papel ético y funciones del médico ante la medicina preventiva y social, entre otros.

《 Con la elaboración de un diario como

una actividad central, se fomentó el hábito

de la escritura y de la observación como

metodología de investigación y ejercicio

etnográfico. 》

Dado que cultivar el arte de escribir se considera una actividad "cognitiva extremadamente compleja" (Nunan, 1991: 36), fomentarlo y revalorarlo empezó a ser una inquietud personal que me llevó a interrogarme " ¿de qué forma podía desplegar el potencial de ingenio artístico, imaginación, conocimiento del estilo personal de escribir y creatividad de mis estudiantes en la composición de textos?".

Recuerdo que en la sesión de encuadre del curso Ensayo científico (EC), los ojos parecieron salirse de sus órbitas al verles la cara de asombro a mis estudiantes del grupo $2^{\circ} \mathrm{B}$ cuando les comenté que uno de los textos finales que elaborarían y presentarían en un plazo de dos meses y medio ante sus compañeros sería una autoetnografía. La asignatura de EC tiene el propósito de conectar al estudiante con el desarrollo histórico de la producción textual científica de la disciplina, conocer la diversidad de sus géneros textuales, relacionar la teoría con la práctica en la elaboración de textos (involucrando aspectos metodológicos, éticos, gramaticales, estructurales, investigativos) y destacar la importancia de la LYEA en la actividad profesional actual.

Para atender las habilidades del estudiante con la escritura, me basé en la propuesta PGA de Badger \& White (2000), para quien el que escribe involucra el conocimiento del lenguaje, el contexto en el que ocurre y las habilidades en su uso, por ello, puede lograrse cuando se extrae el potencial de los aprendices y se les proporcionan los recursos necesarios. El PGA es un enfoque que agrupa algunos elementos clave de aproximaciones tradicionales y alternativas que se han venido utilizando en la enseñanza de idiomas, particularmente (ESL, English as a Second Language); toma del enfoque producto la valoración de los textos modelo; del enfoque proceso retoma la planeación y los borradores; y del enfoque género el conocimiento de los distintos géneros textuales. Es un modelo didáctico circular que en su lado izquierdo ubica diversas etapas relacionadas una a otra en ambos sentidos: Situation (situación), Purpose (propósito), Consideration of mode (consideración de modo), Field (campo), Tenor (tenor), Planning (planea- ción), Drafting (bosquejo), Publishing and Text (edición y texto final); y a su derecha se corresponden con el docente, el estudiante y los materiales de apoyo (Tudor, 2016).

A partir de aquí los cambios curriculares a la asignatura empezaron a implementarse: dividí la clase semanal en dos sesiones, en la primera atendí los temas del programa y en la segunda implementé las actividades del taller de lectura y escritura (TLE); estas últimas se desarrollaron durante todo el curso y se dedicaron por completo al seguimiento, orientación y composición del texto. Iniciamos con algunas lecturas obligatorias tomadas del libro "La cocina de la escritura" de Daniel Cassany (1995): de lo que hay que saber para escribir bien, de las ganas de hacerlo, de lo que se puede escribir, del equipo imprescindible para la escritura y de algunos otros elementos, así como a accionar máquinas, entre otros.

Se seleccionaron como actividades introductorias para que los estudiantes conocieran una parte del arte de escribir, concebir cómo se trabaja el lenguaje en la escritura, cómo los textos son construidos y para entender que un escrito se necesita escribir extensa y recursivamente (Tombro, 2016: 9). Además, hablamos de la importancia del ensayo científico en la disciplina y para el desarrollo profesional futuro de cada uno. Cada semana los estudiantes entregaron ejercicios de escritura (narrativos, descriptivos y argumentativos, entre otros), los cuales eran revisados en cuanto a su estructura, redacción y gramática, y todos se devolvieron a sus autores. Se seleccionaron y fueron expuestos diversos temas por parte de los participantes, a fin de apoyar en la composición del texto: cómo construir párrafos, los conectores discursivos, cómo citar en APA o Vancouver, y la importancia de los signos ortográficos.

Escribir un diario fue una de las actividades centrales del estudiante; su propósito fue crear el hábito de la escritura, fomentar la observación como metodología de investigación y ejercicio etnográfico. Fueron seleccionados diversos textos, con el fin de vincular los contenidos de la unidad de aprendizaje (modos específicos de pensar) con formas particulares de escribir (Carlino, 2012: 21), lo que les ayudaría a relacionar un tópico de investigación con sus experiencias, preocupaciones e intereses (Tombro, 2016: 9). Se dedicaron varias sesiones en su totalidad para que los participantes comentaran sus avances, la estructura de su trabajo, dificultades, soluciones y recomendaciones, a la luz de la lectura de escritos que compañeros de otras generaciones habían realizado. La estrategia central fue la escritura en clase, el avance, el acompañamiento, la plática cara a cara o en pequeños grupos, y la aclaración de dudas; lo curioso fue que la percepción del tiempo fue distinta junto con el espacio de 
clase, además del aula, asimismo, lo fue la sala de lectura y las mesas y bancas de descanso en lugares comunes, en que se combinó con la lectura-revisión y recomendaciones por parte del compañero.

En la sesión final, a cada uno de los participantes se les dieron algunos minutos para que expusieran sus experiencias vividas y entregaron sus textos. Algunos de los títulos autoetnográficos (producto inédito de cada uno de los autores) rescatan esta veta de las vivencias en los espacios clínicos: "Mi relación con pesados recuerdos", "Cimientos y columnas", "Práctica clínica: la base del estudiante médico", "Campos clínicos: el pilar de la medicina", "La paciencia, la mejor medicina", "Del libro al estetoscopio", "Autoetnografía: el sector salud entre frases y experiencias". Una parte de los testimonios que obtuve al final del curso expresaban los siguientes sentimientos y consideraciones: "Este curso me pareció interesante, en momentos resultaba tedioso el hecho de leer algunos artículos que no eran de mi agrado [...]", "Admito que éste no fue mi curso favorito, y lo digo porque redactar no es lo que más me gusta [...]", "Para mí, realizar una autoetnografía fue algo completamente nuevo, no tenía ni idea de cómo hacer una", "[...] es una clase de la cual me tomaba mucho tiempo para la realización de mis trabajos, en ocasiones pensaba que no era de utilidad [...] conforme realizaba mis trabajos [...] podía darme cuenta que mi redacción era mucho mejor que la de antes".

\section{Conclusiones}

El trabajo académico desarrollado desde el enfoque PGA me permitió tener una visión más amplia y darme cuenta de la complejidad del fenómeno de la enseñanza de la escritura y la serie de recursos que necesitamos. Dicho lo anterior, amplié mi radio de acción y centré mi atención en la mejora de las diversas formas en el proceso de adentrar a los estudiantes en la composición de textos académico-científicos. La selección de la autoetnografía me pareció una decisión adecuada, ya que es un texto amigable que despierta el ánimo por la composición de escritos. Creo que a partir de aquí se pueden ir agregando otros géneros textuales y un tiempo razonable en su elaboración, ello dará mayor confianza. Otro aspecto a rescatar son las vivencias grupales, su significado e importancia en la guía y acompañamiento dentro del TLYE, a la vez que atender de manera puntual lo actitudinal-afectivo en la enseñanza de la escritura. Los textos finales -aún con errores- me muestran que propuestas como la presente dan pie para seguir trabajándolas y encontrar eco con la participación de nuestros estudiantes.

\section{Fuentes de consulta}

Badger, R. \& White, G. (2000). A process genre approach to teaching writing. ELT Journal, 54(2), 153-160. Recuperado de: https://www.researchgate.net/ publication/31211657_A_process_genre_approach_to_teaching_writing.

Bochner, A. \& Ellis C. (2016). Evocative Autoethnography. Writing Lives and Telling Stories. New York: Taylor \& Francis Group.

Carlino, P. (2005). La escritura en el nivel superior. En $L a$ Gaceta. Recuperado de: https://bit.ly/2pJeAT4.

Carlino, P. (2012). Escribir, leer y aprender en la universidad. Una introduccion a la alfabetización académica $\left[6^{\mathrm{a}}\right.$. reimp.]. México: Fondo de Cultura Económica.

Cassany, D. (1995). La cocina de la escritura. Barcelona: Anagrama.

Chang, H. (2008). Autoethnography as method. Walnut Creek, CA: Left Coast.

Chartier, Anne-M. (s.f.). El cuaderno de clase: las huellas del cotidiano leer y escribir. Recuperado de: https://bit.ly/2NfRV9V.

Holman, S., Adams, T. \& Ellis, C. (2014). Autoethnography. Understanding Qualitative Research. New York: Oxford University.

Nunan, D. (1991). Language teaching methodology: A textbook for teachers. New York: Prentice Hall.

Pelias, R. (2003). The academic tourist: An autoethnography. Qualitative Inquiry, 9(3), 369-373.

Rusinovci, X. (2015). Teaching Writing Through Process-Genre Based Approach. US-China Education Review A. 5(10), 699-705. Recuperado de: http://www.davidpublisher.org/Public/uploads/ Contribute/564943e786db7.pdf.

Tombro, M. (2016). Teaching autoethnography: Personal writing in the classroom. New York: Open Suny Textbooks.

Tudor, E. (2016). The process genre writing approach; an alternative option for the modern classroom. Disponible en: https://bit.ly/2R3tXzH.

Unidad Académica de Medicina. (2019). Programa de Médico Cirujano. México: uAn. Recuperado en: http://www.uan.edu.mx/es/medico-cirujano. 


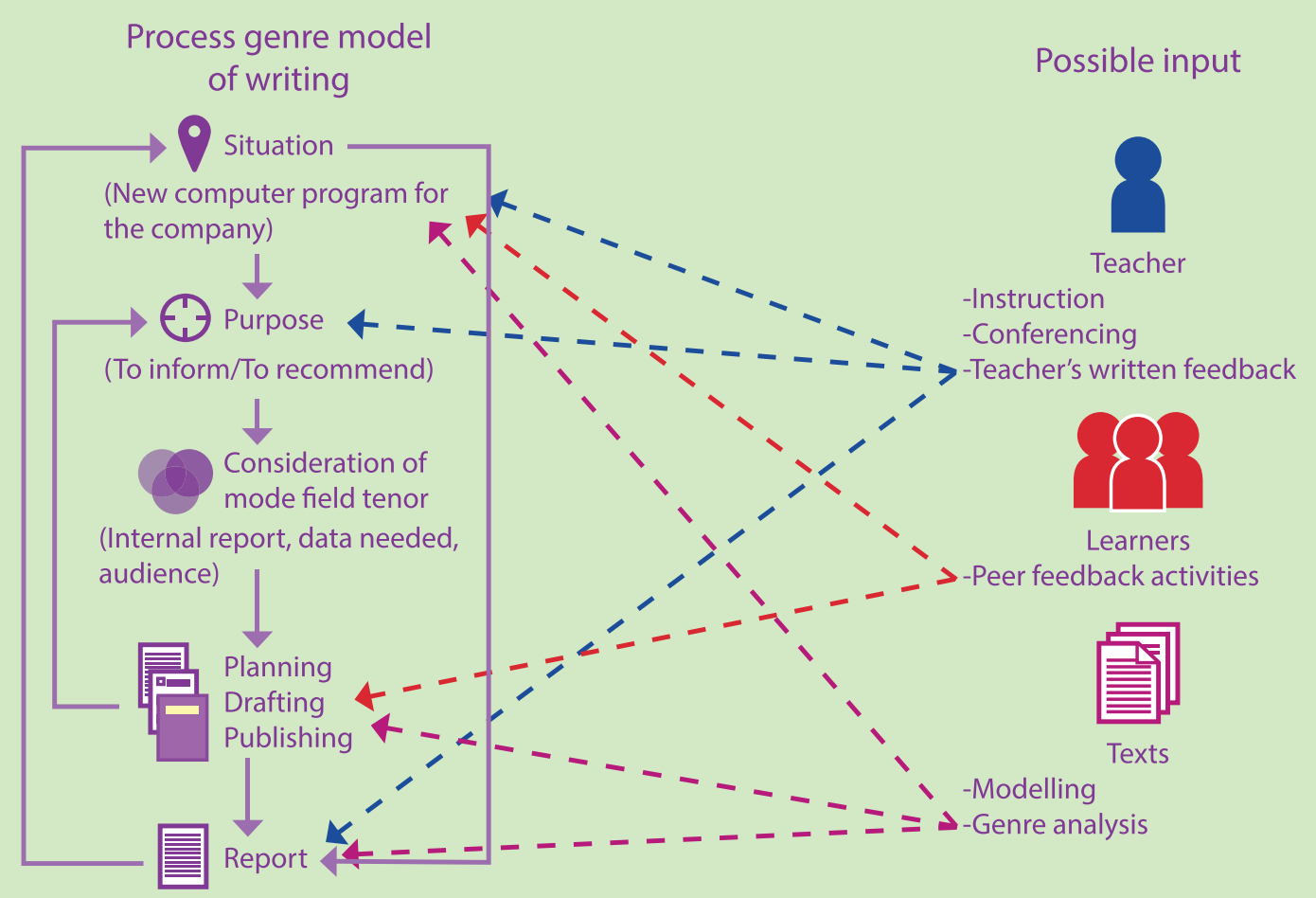

Fuente: Integrated process-genre approach de Badger y White, 2000: 159, citados en Rusinovci, 2015: 702.

De acuerdo con el proceso de Badger and White (2005), retomado por Xhevdet Rusinovci (2015), en la figura se muestra cómo funciona el modelo de una redacción que sigue el método de escritura basado en el proceso y en el género.

La aplicación de este modelo implica que los estudiantes serán concientizados en un primer momento sobre cómo el proceso de escritura sucede en un contexto social y una situación (situation) con el fin de alcanzar un objetivo (purpose).

La siguiente tarea del autor es la consideración de la forma en la que el texto deberá estar construido (mode); el área, campo o disciplina (field) y la elección de palabras de acuerdo a la relación que existe entre el autor y la audiencia a quien el texto está dirigido (tenor).
Una vez definidos estos aspectos sobre la organización del texto, la estructura y el uso del lenguaje, el autor deberá enfocarse en el proceso. Las etapas de revisión y edición son partes integrales del proceso de escritura, y consisten en la planeación (planning), la elaboración, revisión y edición de borradores (drafting) y, finalmente, la publicación de un producto final (publishing).

En este proceso se considera que algunos autores necesitarán de aportes (possible input) de acuerdo al conocimiento que se tenga sobre un género en particular. Estas contribuciones pueden entonces provenir de los facilitadores (teachers), otros estudiantes (learners) y diversos textos o materiales de apoyo (texts). 